\title{
Généalogie des perceptions Est-Ouest
}

\section{Les risques du Cercle Herméneutique ${ }^{l}$}

Zbigniew Truchlewski

\begin{abstract}
Keywords : Central and Eastern Europe, perception, bermeneutic circle

This article deals with perception issues of Western Europe towards Eastern Europe. À vast and abundant literature already tackles this issue, putting forward the mental construction of representations concerning Eastern and Western Europe. Encompassing as it may be, this literature suffers two limits. First, it lacks an analytical framework which would help treating the question rigorously. Second, it does not draw political conclusions of this biased perceptions for the real world. This article uses hermeneutics to offer analytical tools (the comprehension circle in particular) and to understand political crises that the European Union (EU) faced recently. Such an approach gives the possibility to understand the weight of perceptions and the structures of comprehension, and their role in the failures experienced by the EU.

Mots clés : Europe de l'Est, Europe Centrale et Orientale, perception, cercle herméneutique

Cet article fait face aux problèmes des perceptions et des préjugés de l'Ouest à l'égard de l'Est. Ce sujet a déjà été traité dans une littérature de plus en plus abondante, mettant en avant la construction mentale des représentations de l'Europe de l'Ouest et de l'Europe de l'Est. Cette littérature a pourtant deux limites. D'abord, elle ne fournit aucun cadre analytique pour traiter rigoureusement la question. Ensuite, elle ne tire pas les conséquences politiques des perceptions biaisées. Cet article fournit les outils analyti-
\end{abstract}

\footnotetext{
${ }^{1}$ Nous souhaitons remercier chaleureusement les personnes suivantes pour leurs commentaires précieux, tout en les dédouanant des erreurs éventuelles et des opinions de cet article : Volodymyr Kuzka, Quentin Martens, Philippe Perchoc, Arnoldas Pranckevicius, Onorina Savino et Tania Shukan.
} 
ques nécessaires en puisant dans la philosophie herméneutique (le cercle de la compréhension en particulier) et l'applique aux crises politiques récentes qu'a traversées l'Union européenne (UE). Une telle approche permet d'expliquer le poids des préjugés et des structures de compréhension et leur rôle dans les échecs politiques que connait l'UE.

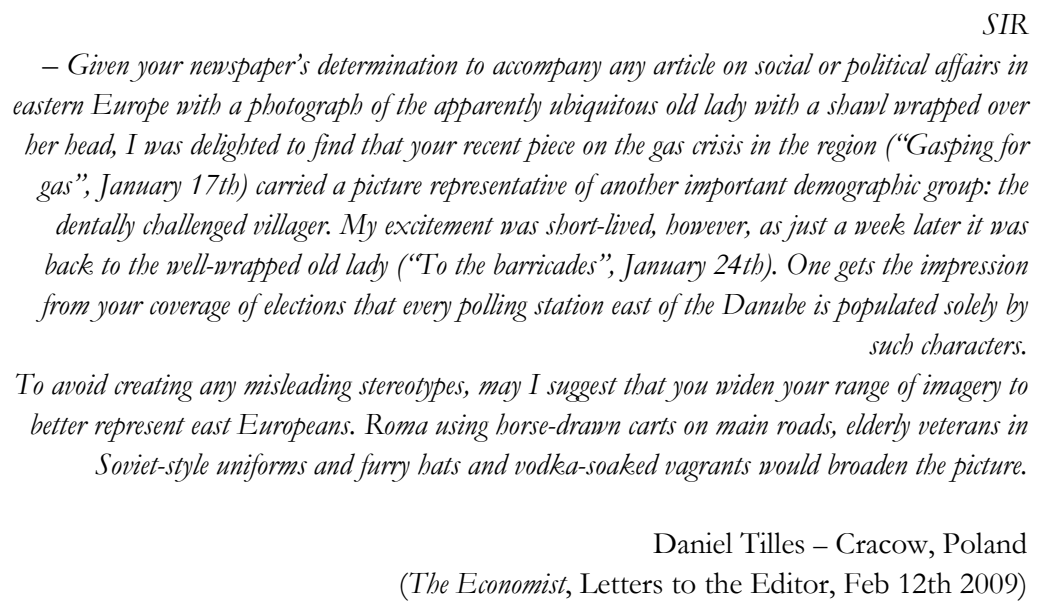

SIR

- Given your newspaper's determination to accompany any article on social or political affairs in eastern Europe with a photograph of the apparently ubiquitous old lady with a shawl wrapped over her head, I was delighted to find that your recent piece on the gas crisis in the region ("Gasping for gas", January 17th) carried a picture representative of another important demographic group: the dentally challenged villager. My excitement was short-lived, however, as just a week later it was back to the well-wrapped old lady ("To the barricades", January 24tb). One gets the impression from your coverage of elections that every polling station east of the Danube is populated solely by such characters.

To avoid creating any misleading stereotypes, may I suggest that you widen your range of imagery to better represent east Europeans. Roma using horse-drawn carts on main roads, elderly veterans in Soviet-style uniforms and furry hats and vodka-soaked vagrants would broaden the picture.

Daniel Tilles - Cracow, Poland (The Economist, Letters to the Editor, Feb 12th 2009)

\section{Introduction}

La question des préjugés a un impact profond sur les relations «Est-Ouest » et a fortiori sur la cohésion politique, économique et sociale dans l'Union européenne (UE). La peur des élargissements successifs a fait voler en éclat l'enthousiasme des années de libération post-communiste. Elle a déçu les nouveaux venus. Les fondements démocratiques de l'UE ont été ébranlés par des élargissements perçus comme arbitraires et contre la volonté des peuples. Celui de 2004 était censé inonder l'Europe de l'Ouest avec des pauvres de l'Est - le plombier polonais - que les citoyens de l'Ouest percevaient rarement comme «européens ", si ce n'est comme des habitants de seconde zone. Il était aussi destiné à paralyser la délicate machinerie institutionnelle. Ces griefs se sont révélés erronés $^{2}$. Ils ont conduit non seulement à un rejet du Traité Constitutionnel européen (Brouard et alii 2007). et à une contestation véhémente de la Directive Bolkenstein,

\footnotetext{
2 Ainsi, comme le montrent Dehousse et alii (2007), la production de la législation communautaire s'est plutôt accélérée après le « Grand Elargissement ». De même, les spectres du plombier polonais et du dumping social hantant l'Europe se sont avérés être une illusion : la Commission européenne a montré (2008) que les migrations en provenance de l'Europe de l'Est n'avaient eu qu'un impact marginal sur les salaires et un impact significativement positif sur la croissance économique.
} 
mais aussi un profond malaise touchant aussi bien l'élargissement, qu'au projet européen et à la place de certains pays dans l'Europe (Lequesne 2008). Il a aussi marginalisé la question de l'acceptation de la Turquie ou des Balkans dans l'UE et grippé les transformations institutionnelles de l'UE. De même, cet élargissement a refroidi les ardeurs des nouveaux membres qui n'ont pas été accueillis dans la «maison européenne» comme ils l'espéraient, ce qui a provoqué, sans doute, un faible taux de participation aux élections européennes de 2004 et 2009.

D’où vient cet échec intellectuel et politique ? Pourquoi persiste-t-il, deux décennies après la chute du mur de Berlin ? Celui-ci ne semble pas s'être effondré dans les esprits et les cartes mentales. D'un côté, l'Europe s'est construite uniquement sur la carte mentale de l'Europe de l'Ouest, jetant aux oubliettes «l'autre Europe » ${ }^{3}$. Mais une fois la guerre froide terminée, les vieilles perceptions ont prévalu. L'Est pleurait de n'avoir pas son plan Marshall, tandis que l'Ouest continuait sur la lancée de l'agenda du Marché Unique et de la Monnaie Unique. Comment en est-t-on arrivé là ? Quels outils utiliser pour analyser cet échec ? L'histoire et l'herméneutique peuvent aider à comprendre la formation des préjugés et à se confronter aux questions de compréhension et d'incompréhension qui minent l'UE.

\section{L'histoire et l'herméneutique : le cercle de la compréhension}

Étudier les pays de l'Est appelle à les comprendre par le biais de nombreuses sciences humaines, de l'histoire à la sociologie, de l'économie à l'ethnologie et aux sciences politiques. Ces sciences ont transmis un savoir qui sert de fondement à notre perception de ces pays. Il s'agit de se demander si ces sciences sociales n'étaient pas elles-mêmes biaisées, si leurs prémisses n'étaient pas en fait des préjugés ou des projections a priori de concepts inadaptés aux réalités des pays étudiés. Cela est important dans la mesure où la transmission de ces recherches et leur enseignement ont pu former des préjugés qui, en dépit de leur lien indubitable avec la réalité étudiée, portaient néanmoins en germe des jugements de valeur déformant la vision de l'objet étudié. Pour comprendre la manière dont l'Ouest a essayé de comprendre l'Est, un outil et une méthode peuvent être fort utiles: l'herméneutique et le cercle de la compréhension. Ces deux éléments s'intéressent simplement à la manière dont les sciences sociales se saisissent de leur objet et montrent la limite de leur connaissance. En quoi consistent ces éléments d'un cadre analytique utile pour comprendre les limites de la connaissance que l'Ouest a de l'Est?

\footnotetext{
${ }^{3}$ L'expression est de Jacques Rupnik (1990). Ce sujet est traité par Norman Davies (2006).
} 
L'herméneutique est une vieille méthode utilisée afin de comprendre les textes anciens et délicats, comme les Écritures Saintes, ou encore les symboles et les mythes. Plus près de nous, des philosophes comme Dilthey (1995 [1900]) ont mis au jour un instrument intellectuel montrant la difficulté de comprendre, à savoir le cercle herméneutique : si l'on veut comprendre un texte, un événement, encore faut-il avoir compris l'œuvre ou le contexte; mais pour comprendre ces derniers, il faut avoir compris les premiers. Le Mur de Berlin s'est effondré ? On ne comprendra pas cet événement sans comprendre le système communiste qui l'a érigé. Mais celui-ci ne se donne à comprendre qu'à travers celui-là. D'autres philosophes ont exploré cette question, comme Heidegger (1986 [1927]) et surtout Gadamer (1996 [1960]). Ils ont mis en évidence un problème propre à toute connaissance: nous approchons tout objet que nous voulons connaître avec « une structure préalable de compréhension », autrement dit, des préjugés ou des perceptions. On peut aussi parler d'anticipations ou de concepts appliqués $a$ priori. Cela revient à regarder un objet à travers des lunettes dont les verres teintés laisseraient voir les formes de l'objet regardé, mais pas la nuance de ses couleurs et les zones grises insaisissables par des dichotomies en noir et blanc. On regarderait ainsi l'apparence sans voir l'essence.

Selon Gadamer, chercher à comprendre, c'est s'exposer aux erreurs suscitées par des préjugés qui n'ont pas été confrontés aux choses elles-mêmes. Mais si pour comprendre des objets on doit faire appel à des préjugés, comprend-on réellement un objet sur lequel nous appliquons nos anticipations, nos préjugés, nos concepts préformés ? Le préjugé, la perception préalable, l'anticipation peuvent aider à comprendre un objet tout comme ils peuvent l'oblitérer et le rendre méconnaissable. Des fausses connaissances et représentations à l'échec pratique dans l'arène politique, il n'y a qu'un pas. Nous agissons en effet d'après des schémas et des préjugés qui nous permettent de juger d'une situation et d'un événement. Sitôt qu'un événement ou qu'une situation ne cadrent pas avec nos préjugés, voilà qu'ils deviennent incompréhensibles. L'exemple le plus frappant est sans doute celui de la chute du Bloc de l'Est qui fut une totale surprise pour les observateurs les plus expérimentés (Comisso 2002, 23). Loin d'exiger des sciences sociales des prédictions impossibles, il faut néanmoins s'interroger sur le regard qu'elles portent sur un objet dont elles n'ont pas vu la réalité. Comment se forment les préjugés ? Gadamer classe les préjugés en deux catégories ${ }^{4}$, les uns étant des préjugés d'autorité, qui nous empêchent d'utiliser notre raison comme il se doit - car l'autorité se substitue au jugement personnel - du fait d'une vérité établie et incontestable (« les pays de l'Est sont sous-développés »), tandis que les seconds, les préjugés de précipitation,

${ }^{4}$ Il faut cependant bien se garder d'avoir des préjugés contre les préjugés ! Ceux-ci aident à comprendre des situations avec des concepts a priori. L'enjeu est donc de distinguer les bons préjugés (ceux qui aident à comprendre) des mauvais (qui déforment la compréhension). 
sont une erreur de la raison (le plombier polonais censé envahir la France). Il y a un lien entre les deux: les préjugés d'autorité informent les préjugés de précipitation. C'est pourquoi il est intéressant de déconstruire ces préjugés afin de découvrir la vision du monde et les représentations dont ils découlent.

La connaissance que l'Europe de l'Ouest a de l'Europe de l'Est dépend en majeure partie de la vision que les historiens en ont donnée. Or, la position des historiens n'est pas sans être biaisée du fait même de leur métier : Raymond Aron (1938) soulignait que nous trouvons dans l'histoire ce que nous y avons introduit nous-mêmes en sélectionnant le donné, la perspective et le point de vue sur la séquence que nous voulons étudier. En ce sens, le jugement de l'historien est historique. ${ }^{5} \mathrm{Il}$ ne faudrait pas pour autant remplacer l'illusion positiviste d'un savoir historique objectif et basculer dans le perspectivisme selon lequel il n'y a pas de fait mais seulement des interprétations, avertit Aron (1938 : 335-362). La question est plutôt de comprendre que «l'extériorité d'un observateur se manifeste dans le choix d'un système et d'une interprétation à l'intérieur d'un certain système. L'historicité d'un historien s'exprime dans le point de vue du spectateur, aussi bien que dans la perspective sur le devenir» (Aron, 1938: 105). De fait, «toute pensée de la société et de l'histoire appartient elle-même à la société et à l'histoire» (Castoriadis 1999). Ce qu'il faut comprendre, par conséquent, c'est la manière dont notre jugement historique sur l'Europe centrale s'est informé.

\section{Généalogie des préjugés de l'Ouest sur l'Est}

\subsection{Les cartes mentales de l'Est}

D’où viennent ces préjugés à partir desquels l'Ouest a essayé de comprendre l'Est et, surtout, pourquoi ont-ils échoué ? On peut trouver des clefs de lecture utile dans la notion de cartes mentales. Celles-ci sont des projections ou préjugés fondés sur des opinions qui se présentent comme évidentes. La sculpture Entropa de la Présidence tchèque de l'UE en 2008 est un exemple de ces cartes mentales: on a pu y voir nombre de préjugés sur toutes les populations de l'UE. Des Lituaniens dans une posture antirusse aux toilettes turques de la Bulgarie en passant par une France en grève, tous les clichés européens ont été mis à nu. C'était montrer notre manière de penser les autres peuples européens en fonction de nos préjugés, de nos «structures de compréhension ».

Les cartes mentales sont une géographie «flottante ». Contrairement à la géographie objective, les cartes mentales sont disproportionnées et relatives aux valeurs dont elles

\footnotetext{
${ }^{5}$ Cela s'applique aussi à ce texte.
} 
découlent. Les représentations de l'espace sont une construction sociale. Ces images cognitives mettent en lumière les relations politiques implicites, les rapports de force et la manière dont ils sont exercés. Le choix des cartes mentales, conscient ou inconscient, avalise et rend légitime une certaine forme de domination ou d'autorité. Elles projettent des valeurs sur la géographie, exacerbent nos perceptions et oblitèrent ce qui importe peu. Une carte mentale du monde faite selon l'importance ou la puissance des pays verrait des États-Unis incroyablement plus grands que le continent africain, alors que celuici l'emporte sur ceux-là par sa taille et sa population. Il en est de même en Europe. Il y a comme un trou noir sur la carte mentale européenne entre Berlin et Moscou. On peine à nommer les dix nouveaux membres de l'UE et à distinguer les pays baltes entre eux. Idem pour les Balkans et le Caucase. Quant à la Russie, elle reste ce vaste empire, à la fois attirant et terrifiant. Mais ce ne sont là que des représentations qui, si elles ont la réalité pour origine, n'en vivent pas moins une existence indépendante de celle-ci.

Plusieurs niveaux d'analyse sont nécessaires pour faire la généalogie de ces perceptions et de ces préjugés. Un premier niveau d'analyse est celui des sciences sociales. En fin historien, Norman Davies (2006) détricote les fils qui font la trame de l'enseignement de l'histoire tel que les Occidentaux le connaissent. Il démonte tous les mythes et les mythologies accumulés durant les siècles et qui reflétaient autant les préjugés des historiens que les rapports de force de l'époque et montre comment la sédimentation des préjugés a résulté en des perceptions tenaces. Davies montre d'abord les faux contrastes sur lesquelles est fondée l'histoire telle qu'elle est enseignée et les comparaisons qu'il faudrait faire pour rendre justice à l'histoire telle qu'elle a été. Dans la première catégorie, on peut mettre les projections des savants de l'Ouest. Certains d'entre eux ont fait des études de villages slaves en se fondant uniquement sur un village de Russie, le distinguant clairement des villages occidentaux. Comme si les villages situés entre Berlin et Vladivostok étaient identiques. Maria Todorova (1991) a montré que rien n'était plus faux : s'en prenant à une démarche identique dans les Balkans, elle y montre l'hétérogénéité des formes d'organisation sociale. Cette méthode, en plus d'être réductionniste, postule avec force que tous les pays inconnus sont en fait les mêmes: ils sont une périphérie. Les termes et les concepts utilisés par l'Ouest sont biaisés depuis longtemps. Les Grecs et les Romains avaient déjà stigmatisé la figure de l'autre en le désignant comme "Barbare ». Cela revenait à rejeter une altérité qui aurait pu être féconde. Hall (1989) remarque cependant que cela servait à justifier une conquête de l'identité en opposition à un autre dont les Grecs puis les Romains ont cherché à se démarquer. La civilisation européenne de la Renaissance puis la civilisation Occidentale ont repris ce modèle excluant l'altérité. Tout cela se passe au moment où les Empires d'Europe centrale se délitent et laissent place à une Europe de la Renaissance, des Lumières puis de l'industrialisation, du capitalisme et de l'État-Nation.

Ce biais est évident des deux côtés du rideau de fer qui fissure nos cartes mentales. À l'Ouest, Kulczycki (2005) montre de manière convaincante, bibliographie à l'appui, que l'idée de civilisation occidentale est un artefact reflétant moins une vérité historique que 
le rapport de force qui a présidé à son émergence. L'étude de la civilisation occidentale n'est en effet concentrée que sur les grandes puissances qu'ont été la France, la GrandeBretagne et l'Allemagne. Quant à l'Est, Willberger (1972) et Wolff (1994) surtout ont mis en évidence la catégorie mentale «Europe de l'Est», devenue convenable pour étiqueter un terrain historique et géographique méconnu par l'Occident. L’exemple typique est celui de Voltaire qui, dans son Histoire de Charles XII, découvre une Russie qu'il loue sans y être jamais allé et se gausse des peuples d'Europe centrale sans mieux les connaître. Norman Davies (2006) remarque que la guerre froide a renforcé la catégorie mentale d'Europe de l'Est, désignée comme l'autre, l'ennemi de la civilisation occidentale. Des années 1950 aux années 1970, 70\% de la littérature consacrée à l'Europe de l'Est ne traitait que de la Russie ou de l'URSS. L'entre-deux autrement appelé Europe centrale est resté un trou noir intellectuel sur les cartes mentales de l'Occident.

Le concept d'Europe centrale et orientale est polysémique. Il n'a aucune signification précise parce qu'il a été utilisé dans des contextes différents, formulé de manière différente et à différents desseins. La cartographie des cartes mentales de l'Est (voir les tableaux 1 et 3) montre ses significations dépendantes du contexte politique et géopolitique et de la langue dans laquelle il est énoncé. Plusieurs désignations qualifient ce territoire aux contours flous: Europe centrale, Europe centrale et orientale, Europe médiane, Mitteleuropa, Ostmitteleuropa, Europe de l'Est. Cette région est en fait un palimpseste géographique constitué de discontinuités historiques. Sa conceptualisation est historique et dynamique et ne peut se réduire à un concept spatial statique. L'Europe centrale (Garton Ash, 1999) est contingente du contexte politique - on peut décider d'y inclure la Slovaquie si celle-ci est démocratique. C'est aussi un concept géographique extrêmement souple - où sont ses frontières ? L'Europe centrale et orientale désigne dans un même concept deux Europe différentes, regroupant les Slaves de l'Est et les Slaves de l'Ouest, postulant une identité commune pour un ensemble scindé en deux par le Schisme de 1054 et la frontière de l'ancienne URSS. L'Europe médiane est un artefact géographique, compromis des réflexions des géographes désireux de définir de manière objective un ensemble incohérent du point de vue historique mais qui, au moment où les géographes le forgeaient, traversait les mêmes épreuves. Désignant la même chose, les termes d'Europe centrale et de Mitteleuropa ont une signification diamétralement opposée. Domination germano-autrichienne pour cette dernière et, pour la première, indépendance des petites nations désireuses de s'affranchir du joug germano-autrichien et de se distancier d'une Europe de l'Est sous les fourches caudines de la Russie. 
Tableau 1. Typologie des cartes mentales de l'Europe centrale ${ }^{6}$

\begin{tabular}{|c|c|c|c|c|}
\hline $\begin{array}{l}\text { Concep- } \\
\text { tion }\end{array}$ & Nom(s) & $\begin{array}{l}\text { Filtres sous- } \\
\text { jacents }\end{array}$ & $\begin{array}{l}\text { Vision Géopoli- } \\
\text { tique }\end{array}$ & Evolution \\
\hline Allemande & $\begin{array}{l}\text { Mittel- } \\
\text { europa } \\
\text { (apparu } \\
\text { vers } 1840, \\
\text { discrédité } \\
\text { après 1945) }\end{array}$ & $\begin{array}{l}\text { L'Allemagne est } \\
\text { inclue dans cette } \\
\text { Mitteleuropa; Drang } \\
\text { nach Osten, pan- } \\
\text { germanisme, impé- } \\
\text { rialisme }\end{array}$ & $\begin{array}{l}\text { Construction d'un } \\
\text { centre fort entre la } \\
\text { France et la Rus- } \\
\text { sie, inclusion de } \\
\text { l'Autriche }\end{array}$ & $\begin{array}{l}\text { Zentraleuropa, Zwis- } \\
\text { cheneuropa, Ostfors- } \\
\text { chung, Ostmittel- } \\
\text { europaforschung' An- } \\
\text { nées } 1960 \text { : de } \\
\text { l'espace allemand à } \\
\text { la diversité intrin- } \\
\text { sèque d'un espace } \\
\text { voisin }\end{array}$ \\
\hline Autricbienne & $\begin{array}{l}\text { Mittel- } \\
\text { europa }\end{array}$ & $\begin{array}{l}\text { Pont entre Orient } \\
\text { et Occident, nos- } \\
\text { talgie habsbour- } \\
\text { geoise, zone } \\
\text { d'influence éco- } \\
\text { nomique, fédéra- } \\
\text { lisme, respect des } \\
\text { nations }\end{array}$ & $\begin{array}{l}\text { Contre la Russie } \\
\text { (barrer la route } \\
\text { des détroits), } \\
\text { concurrence avec } \\
\text { l'Allemagne en Eu- } \\
\text { rope de l'Est, dé- } \\
\text { laissée pour les } \\
\text { Balkans }\end{array}$ & $\begin{array}{l}\text { De la rivalité avec } \\
\text { l'Allemagne, } \\
\text { l'antagonisme russe } \\
\text { et nostalgie vers } \\
\text { un concept ouvert } \\
\text { contribuant à } \\
\text { l'identité autri- } \\
\text { chienne }\end{array}$ \\
\hline Russe $e^{10}$ & $\begin{array}{l}\text { Europe } \\
\text { Centrale }\end{array}$ & $\begin{array}{l}\text { Panslavisme russo- } \\
\text { centré } 11 \text {, expan- } \\
\text { sionnisme, messia- } \\
\text { nisme }\end{array}$ & $\begin{array}{l}\text { Fédération russo- } \\
\text { centrée dirigée } \\
\text { contre l'Empire } \\
\text { Ottoman, la Prusse } \\
\text { et l'Autriche- } \\
\text { Hongrie }\end{array}$ & $\begin{array}{l}\text { Eurasie, "maison } \\
\text { commune euro- } \\
\text { péenne" }\end{array}$ \\
\hline Française 12 & $\begin{array}{l}\text { Europe } \\
\text { Centrale, } \\
\text { Europe de }\end{array}$ & $\begin{array}{l}\text { Prisme de grande } \\
\text { puissance éclipse } \\
\text { les petites nations, } \\
\text { biais des média- }\end{array}$ & $\begin{array}{l}\text { Attitude ambiva- } \\
\text { lente vis-à-vis de } \\
\text { la Russie peu } \\
\text { compatible avec }\end{array}$ & $\begin{array}{l}\text { Vision schématique } \\
\text { soit binaire (Est et } \\
\text { Ouest) soit étati- } \\
\text { que (prisme des }\end{array}$ \\
\hline
\end{tabular}

${ }^{6}$ Adapté de Horel (2009). Ces cartes mentales sont purement indicatives. Elles évoluent dans le temps et en fonction de la situation politique. Elles renseignent plus sur les impératifs stratégiques que sur la région ellemême.

${ }^{7}$ Meyer Cord Henry (1995).

${ }^{8}$ Respectivement Europe centrale, Europe « entre », recherche sur l'est et recherche sur le centre est.

${ }^{9}$ Le concept de Mitteleuropa est polysémique : outre l'Allemagne et l'Autriche, il a aussi été utilisé par les anti-communistes dans les années 1980 pour se dégager du joug soviétique, par ceux qui y voyaient une alternative à la «maison commune » de Gorbatchev, une politique de voisinage avant l'heure vu les incertitudes après la chute du communisme ou encore une invitation à l'ouverture et à l'échange culturels.

${ }^{10}$ Dmitrieva (1994).

11 À différencier par exemple de l'austroslavisme marqué par les révolutions de 1848, du néoslavisme dérivé de la révolution russe de 1905 (séparé de la notion d'impérialisme) ou du slavisme polonais. Le panslavisme a aussi été associé au communisme et à l'anti-occidentalisme, de Herzen ou Bakounine. Le slavisme russe a été influencé par des polonais comme le prince Adam Czartoryski.

12 Marès (1991). 


\begin{tabular}{|c|c|c|c|c|}
\hline & l'Est & $\begin{array}{l}\text { teurs (émigration), } \\
\text { facteur allemand }\end{array}$ & $\begin{array}{l}\text { le soutien aux na- } \\
\text { tions d'Europe } \\
\text { centrale; contre- } \\
\text { poids à } \\
\text { l'Allemagne }\end{array}$ & $\begin{array}{l}\text { traités de paix) qui } \\
\text { rend illisible } \\
\text { l'Europe centrale }\end{array}$ \\
\hline Italienne & $\begin{array}{l}\text { Europe } \\
\text { Centrale et } \\
\text { Orientale }\end{array}$ & $\begin{array}{l}\text { Europe centrale } \\
\text { des nations, cen- } \\
\text { trée sur la Hongrie } \\
\text { et le Danube } \\
\text { (prisme du "Prin- } \\
\text { temps de peu- } \\
\text { ple»s) }\end{array}$ & $\begin{array}{l}\text { Signification plus } \\
\text { culturelle que géo- } \\
\text { politique, moins } \\
\text { important que les } \\
\text { Balkans mais peut } \\
\text { servir de contre- } \\
\text { poids à } \\
\text { l'Allemagne et } \\
\text { l'Autriche-Hongrie }\end{array}$ & $\begin{array}{l}\text { Vers une vision } \\
\text { large de l'Europe } \\
\text { centrale, jusqu'à la } \\
\text { Russie et les pays } \\
\text { baltes }\end{array}$ \\
\hline Anglaise & $\begin{array}{l}\text { Europe } \\
\text { centrale - } \\
\text { Europe de } \\
\text { l'Est }\end{array}$ & $\begin{array}{l}\text { Différentiation des } \\
\text { deux Europe (cen- } \\
\text { trale et Est) pour } \\
\text { marquer une diffé- } \\
\text { rence avec l'URSS } \\
\text { puis la Russie, } \\
\text { l'Ukraine, le Bela- } \\
\text { rus. }\end{array}$ & $\begin{array}{l}\text { Zone de compéti- } \\
\text { tion entre les puis- } \\
\text { sances continenta- } \\
\text { les (Allemagne, } \\
\text { Autriche-Hongrie, } \\
\text { Russie, France) }\end{array}$ & $\begin{array}{l}\text { Vers une plus } \\
\text { grande reconnais- } \\
\text { sance de la spécifi- } \\
\text { cité de la région } \\
\text { sans toutefois se } \\
\text { départir d'une vi- } \\
\text { sion de bloc }\end{array}$ \\
\hline Américaine & $\begin{array}{l}\text { Europe de } \\
\text { l'Est }\end{array}$ & $\begin{array}{l}\text { Filtre Wilsonien } \\
\text { d'autodétermin- } \\
\text { ation des peuples, } \\
\text { de la propagation } \\
\text { de la démocratie }\end{array}$ & $\begin{array}{l}\text { Zone à utiliser } \\
\text { comme carte } \\
\text { contre l'URSS, la } \\
\text { Russie ou dans la } \\
\text { construction euro- } \\
\text { péenne }\end{array}$ & $\begin{array}{l}\text { Entre non- } \\
\text { engagement, isola- } \\
\text { tionisme et straté- } \\
\text { gie de "contain- } \\
\text { ment" (Bush: } \\
\text { missiles et radar: } \\
\text { Obama: désenga- } \\
\text { gement) }\end{array}$ \\
\hline
\end{tabular}

Les mêmes préjugés négatifs sont au fondement d'idées concernant les Balkans (le terme de « balkanisation » est péjoratif - pourquoi ne pas utiliser fragmentation ? - tout comme celui de "poudrière balkanique ») et l'Orient. Mais ces deux idées ont droit à un traitement différent, comme le souligne Maria Todorova (1997). Le balkanisme n'est pas un orientalisme. Les Balkans renvoient à une notion et à un territoire concret, tandis que les frontières de l'Orient sont floues et imaginaires. Les Balkans sont un concept de transition, dont on ne peut pas dire qu'il soit non européen, de même qu'on ne peut pas dire que les Balkans ont été colonisés. Surtout, et curieusement pour les Balkans, ils ne symbolisent pas l'Islam que contient l'Orient. Et, enfin, les Balkans ne sont pas catégorisés comme une race à part, différente de l'homme blanc européen. Ils ont une identité qui leur est propre et qui, au demeurant, est aussi créée contre cet autre symbolisé par l'Orient et incarné par l'Empire ottoman et turc. 


\subsection{Une géographie « flottante»}

Un autre niveau d'analyse permet de montrer que les sciences sociales ont déteint sur les médias et les préjugés populaires. L'exemple le plus frappant est sans doute celui de la bande dessinée en général et de Tintin en particulier. La géographie des aventures du reporter du Petit Vingtième l'amène en terrain imaginaire dans le cas du Sceptre d'Ottokar (1939) et de L'Affaire Tournesol (1956). Deux pays y sont évoqués, qui symbolisent la manière dont on se représente l'Est plus de cinquante ans après la parution de ces bandes dessinées. La Syldavie, mélange de Transylvanie et de Moldavie, évoque les Balkans et la domination des Empires austro-hongrois et ottoman sur une contrée "sousdéveloppée ", au pied des Zmylhlpathes (Carpathes). La Bordurie symbolise quant à elle les régimes autoritaires et totalitaires, fascistes aussi bien que communistes (le dictateur Plekszy-Gladz, salué d'un Amaï à consonance nazie, porte la moustache de Staline). Surtout, c'est le nom même de Bordurie qui retient l'attention. Il évoque en effet le terme de «frontière » qui implique une différentiation par rapport au monde dans lequel évolue le protagoniste. La Bordurie et la Syldavie sont en effet cet « autre » déprécié et qui incarne le parent pauvre de la civilisation occidentale. En témoigne les attributs quelque peu ridicules de ces pays : hormis le fait qu'ils sont peuplés de paysans et que leur régimes politiques sont aux antipodes des démocraties progressistes de l'Ouest, le langage qu'ont leur attribue contribue à les aliéner. Il emprunte sa forme à l'alphabet cyrillique et, qui plus est, son contenu est dévalué (la devise syldave, Eih bennek, eih blavek, est traduite par « qui s'y frotte s'y pique »). Le fait qu'Hergé rende ces territoires imaginaires, tout en les renvoyant à une base réelle mais non-définie, contribue à mettre sur le regard des lecteurs un voile d'ignorance. Ce voile d'ignorance empêche de voir la réalité complexe de ces pays. Ce n'est qu'un exemple parmi d'autres, mais des plus significatifs. Comment peut-on dès lors s'étonner des préjugés dont les pays de l'Est sont victimes? En ce sens, «le problème semble résider dans le fait l'Europe centrale court toujours le risque d'être le produit de notre imagination » (Judt 1991).

Les concepts utilisés pour désigner l'autre Europe sont donc non seulement historiques et politiques avant d'être rigoureusement géographiques, mais en plus ils participent d'une logique de sédimentation. Reposant les uns sur les autres comme un palimpseste inextricable, ils favorisent un traitement imaginaire et par le biais de préjugés. Comme le montre Davies (2001 : 458-460), cela n'est aucunement facilité par le fait que, d'une part les noms est-européens sont complexes, et que d'autre part les travaux des historiens sont souvent inexacts. Plusieurs biais entrent en jeu : le biais historique et l'origine des historiens (un historien russe ne donnera pas les mêmes noms que des historiens allemands, anglais ou français - voir tableau 2), une mauvaise traduction d'un atlas publié dans une langue étrangère qui mène à l'anachronisme (ainsi, les auteurs anglais du Times Atlas of World History ont utilisé des noms russes et allemands du dix-neuvième siècle pour des cartes médiévales montrant des endroits n'ayant à l'époque aucun rapport avec la Russie ou l'Allemagne ou 
bien encore tel atlas anglais, version d'un ancien atlas allemand, qui a traduit le texte allemand en anglais mais pas les noms des lieux), le manque de précision historique qui mettrait les noms est-europeéns dans leur contexte temporel, spatial et d'usage. La question qu'il faut se poser en étudiant la géographie de l'Europe de l'Est, c'est quand, où, pourquoi, et par qui le nom de l'endroit qui nous intéresse a été utilisé.

\section{Tableau 2. L'Est, une géographie flottante ${ }^{13}$}

\begin{tabular}{|c|c|c|c|c|}
\hline Français & Anglais & Allemand & Russe & Ukrainien \\
\hline Vilnius & Vilnius & Wilna & Vil'nius & Vil'nius \\
\hline Lvov/Lviv & Lviv & Lemberg & L'vov & L'viv \\
\hline Kiev & Kyiv & Kiew & Kiev & Kyiv \\
\hline Minsk & Minsk & Minsk & Minsk & Mins'k \\
\hline Galicie & Galicia & Galizien & Galitsiia & Halychyna \\
\hline Volinie & Volhynia & Wolynien & Volyn' & Volyn' \\
\hline Pologne & Poland & Polen & Pol'sha & Pol'shcha \\
\hline Lituanie & Lithuania & Litauen & Litva & Lytva \\
\hline Biélorussie & Belarus & Weissrussland & Belorussija & Bilorus' \\
\hline Ukraine & Ukraine & Ukraine & Ukraina & Ukraina \\
\hline Russie & Russia & Russland & Rossiia & Rosiia \\
\hline Polonais & Biélorusse & Yiddish & Lithuanien & \\
\hline Wilno & Vil'nia & Vilne & Vilnius & \\
\hline Lwów & L'vou & Lemberik & Lvovas & \\
\hline Kijów & Kieu & Kiv & Kijevas & \\
\hline Mińsk & Miensk & Minsk & Minskas & \\
\hline
\end{tabular}

${ }^{13}$ Source : compilation personnelle à partir de Snyder (2003), Davies (2001) et Zaprudnik (1993). 


\begin{tabular}{|c|c|c|c|}
\hline Galicja & Halitsiia & Galitsye & Galicija \\
\hline Wołyń & Valyn & Volin & Volyne \\
\hline Polska & Polshcha & Poyln & Lenkija \\
\hline Litwa & Letuva & Lite & Lietuva \\
\hline Białoruś & Belarus' & Vaysrusland & Baltarusija \\
\hline Ukraina & Ukraina & Ukraine & Ukraina \\
\hline Rosja & Ras'eia & Rusland & Rusja \\
\hline
\end{tabular}

Davies dénombre quatre catégories de noms et d'erreurs qui ont pu prêter à confusion : 1/ les noms de lieux locaux utilisant le langage indigène, ce qui est compliqué par le fait que les lieux d'Europe de l'Est sont souvent multiculturels (voir, par exemple, le cas de Vilnius) ; 2/ les noms de lieux officiels qui découlent de la langue de l'autorité en place et changent en même temps que celle-ci (ainsi la même ville peut s'appeler Wrastilaw avant 1335 en sus de son nom latin Vratislavia, Vraclav de 1335 à 1526, Breslau de 1526 à 1945 et Wrocław depuis 1945); 3 / les noms de lieux politiques (Stanisławów, du nom du père de Andrzej Potocki, qui avait acheté le village, est ensuite devenu Ivanofrankovsk en mémoire du poète ukrainien Ivan Franko, ou encore Stalingrad maintenant Volgograd et Saint-Pétersbourg autrefois Leninegrad) dont l'exemple le plus parlant est sans doute celui du transfert des capitales (Fauve 2007); 4/ les noms de lieux traduits, adaptés d'une autre langue (l'aspect le plus grave étant la traduction du cyrillique, car il faut transcrire en alphabet latin et dans des langues différentes des sons ou des lettres inexistants, comme «щ», «ц» ou «ч»), ou historiquement censurés (par exemple, en ce qui concerne la Pologne, les Anglais utilisent des noms allemands adaptés - Warschau devient Warsaw - tandis que les Français et les Italiens usent des noms latins - Varsovia devient Varsovie). Tous ces biais démultiplient le nombre de versions du nom d'un lieu, ce qui ajoute à la confusion (voir tableau 2). On voit ainsi émerger le concept d'une géographie «flottante », se déplaçant au gré des valeurs et des préjugés, où se superposent la géographie, l'histoire, la politique, les perceptions et les erreurs voulues ou non.

\subsection{Le paradoxe européen}

La généalogie des préjugés qui vient d'être esquissée montre surtout autre chose (voir tableau 3 ci-dessous). Ce qui est frappant, c'est sans aucun doute leur structure « exclusive ». Loin de poser une inclusion de l'autre, l'Ouest s'est défini inconsciemment en rejetant ce qu'il ne percevait pas comme lui-même - l'Est. L'autre est par essence inférieur. Cela est d'autant plus frappant que cela se fait souvent au moment où l'Ouest se 
fait le héraut des valeurs dites « universelles ». Il y a là comme un paradoxe : ces valeurs prouveraient en fait la supériorité de l'artefact historique qui les érige en loi indiscutable, jetant les fondements d'une idéologie coloniale et impériale.

L'enjeu n'est pas de faire le procès d'une pensée exclusive. Bien plutôt s'agit-il de montrer que l'on se trouve devant une contradiction fondamentale de la construction européenne. Alors que celle-ci est fondée sur des concepts inclusifs, elle doit néanmoins se construire sur des concepts fondamentalement opposés. Émerge ici une dissonance cognitive, entre un impératif catégorique à la base de la construction européenne professant l'unité du peuple européen, et des concepts renvoyant dos à dos deux unités, l'Ouest et l'Est.

N'est-il pas alors contradictoire de penser l'élargissement en ces termes et impossible de penser une éventuelle identité européenne? 
Tableau 3. Cartes mentales de «l'Est » : une cartographie

\begin{tabular}{|c|c|c|c|}
\hline Cartographie & Idées sous-jacentes & Origine/époque & Aspect politique \\
\hline Les Barbares $^{14}$ & Rejet de ce qui est différent & Antiquité & Exclusion de l'autre \\
\hline $\begin{array}{l}\text { Civilisation euro- } \\
\text { péenne }\end{array}$ & $\begin{array}{l}\text { Italie et France centres de la } \\
\text { civilisation européenne }\end{array}$ & Renaissance $^{15}$ & $\begin{array}{l}\text { Création du préjugé d'un } \\
\text { Est inférieur et non civilisé }\end{array}$ \\
\hline $\begin{array}{l}\text { Europe/ } \\
\text { Civilisation occi- } \\
\text { dentale }^{16}\end{array}$ & $\begin{array}{l}\text { Concert des nations, histoire } \\
\text { européenne vue en termes de } \\
\text { relations internationales ex- } \\
\text { cluant de facto les non nations } \\
\text { ou les non-États }\end{array}$ & $\begin{array}{l}\text { Siècle des Lumières } \\
\text { Dix-neuvième siècle }\end{array}$ & $\begin{array}{l}\text { Ouest et Est sont deux enti- } \\
\text { tés distinctes, la première } \\
\text { étant européenne et supé- } \\
\text { rieure. Impérialisme occi- } \\
\text { dental et russe. }\end{array}$ \\
\hline \multirow{3}{*}{$\begin{array}{l}\text { Europe centrale }^{17} \\
\text { (vision intérieure et } \\
\text { non plus exté- } \\
\text { rieure) }\end{array}$} & $\begin{array}{l}\text { Tomáš Masaryk : petits États } \\
\text { indépendants de toute domi- } \\
\text { nation allemande, autrichienne } \\
\text { ou russe }\end{array}$ & $\begin{array}{l}\text { Première guerre mon- } \\
\text { diale }\end{array}$ & $\begin{array}{l}\text { Volonté de s'émanciper et } \\
\text { de recréer les États-nations } \\
\text { d'Europe centrale }\end{array}$ \\
\hline & $\begin{array}{l}\text { Anti-soviétique, émancipatoire } \\
\text { et identité nationale }{ }^{18}\end{array}$ & Guerre froide & $\begin{array}{l}\text { Démarcation de l'URSS et } \\
\text { de l'« asiatisme barbare » }\end{array}$ \\
\hline & $\begin{array}{l}\text { Pays d'Europe centrale veu- } \\
\text { lent se distinguer des Bal- } \\
\text { kans }^{19} \text { et de la CEI }{ }^{20}\end{array}$ & Années 1990 & $\begin{array}{l}\text { Intégration rapide dans } \\
\text { l'UE; racines démocratiques. }\end{array}$ \\
\hline Mitteleurop $\mathrm{a}^{21}$ & $\begin{array}{l}\text { Domination germano- } \\
\text { autrichienne }\end{array}$ & $\begin{array}{l}\text { Première guerre mon- } \\
\text { diale }\end{array}$ & $\begin{array}{l}\text { Traiter ces pays comme des } \\
\text { objets et non des sujets des } \\
\text { relations internationales }\end{array}$ \\
\hline Europe médiane ${ }^{22}$ & $\begin{array}{l}\text { Europe non soviétique aupa- } \\
\text { ravant sous domination com- }\end{array}$ & 1990 à nos jours & $\begin{array}{l}\text { Artefact des géographes } \\
\text { pour penser un entre-deux }\end{array}$ \\
\hline
\end{tabular}

${ }^{14}$ Hall (1989).

${ }^{15}$ Wolff (2001a et b)

${ }^{16}$ Kulczycki (2005)

${ }^{17}$ Sinnhuber (1954) a identifié seize définitions différentes de l'Europe centrale.

${ }^{18}$ Kundera (1984) et Garton Ash (1999).

${ }^{19}$ Godsworthy (1998).

${ }^{20}$ Konrad (1994).

${ }^{21}$ Naumann (1915).

${ }^{22}$ Lacoste (1988), Michel (1997) et Zrinscak (1999), repris ensuite par l'INALCO. 


\begin{tabular}{|c|c|c|c|}
\hline & muniste & & en transition \\
\hline \multirow{3}{*}{$\begin{array}{l}\text { Europe de l'Est } \\
\text { (Europe orientale) }\end{array}$} & $\begin{array}{l}\text { Vide géopolitique entre Berlin } \\
\text { et Moscou caractérisé en ter- } \\
\text { mes d'infériorité }\end{array}$ & $\begin{array}{l}\text { Siècle des Lumières }{ }^{23} \text {, } \\
\text { déclin des puissances } \\
\text { d'Europe centrale }\end{array}$ & $\begin{array}{l}\text { Idée de ce qui est européen } \\
\text { et de ce qui l'est beaucoup } \\
\text { moins (l'Est) }\end{array}$ \\
\hline & $\begin{array}{l}\text { Tout ce qui n'est pas } \\
\text { le Bloc de l'Ouest }\end{array}$ & Guerre froide ${ }^{24}$ & $\begin{array}{l}\text { Légitimation de l'ordre de } \\
\text { Yalta }^{25} \text {; l'intérêt de certains } \\
\text { groupes occidentaux }^{26}\end{array}$ \\
\hline & $\begin{array}{l}\text { Différentiation de l'Europe de } \\
\text { l'Ouest et du Centre avec } \\
\text { pour fracture l'ancienne URSS } \\
\text { et la religion orthodoxe mais } \\
\text { aussi la corruption et la vio- } \\
\text { lence }\end{array}$ & Années 1990 & $\begin{array}{l}\text { Rejet d'un Est non euro- } \\
\text { péen, presque asiatique }\end{array}$ \\
\hline $\begin{array}{l}\text { Balkans, balka- } \\
\text { nisme balkanisa- } \\
\text { tion } 27\end{array}$ & $\begin{array}{l}\text { Catégories négatives désignant } \\
\text { l'instabilité politique, la vio- } \\
\text { lence et la fragmentation poli- } \\
\text { tique }\end{array}$ & $\begin{array}{l}\text { Fin du XIXe }{ }^{\mathrm{e}} \text {, début et fin } \\
\text { du } \mathrm{XX}^{\mathrm{e}}\end{array}$ & $\begin{array}{l}\text { Désintéressement des Bal- } \\
\text { kans, banalisation de la } \\
\text { guerre, idée de retard }\end{array}$ \\
\hline $\begin{array}{l}\text { Orient et orienta- } \\
\text { lisme } \mathrm{e}^{28}\end{array}$ & $\begin{array}{l}\text { L'Islam et l'Asie comme Au- } \\
\text { tre, racisme, construction } \\
\text { imaginaire d'un alter ego de } \\
\text { l'Occident. }\end{array}$ & $\begin{array}{l}\text { Expansion coloniale du } \\
\text { XIX } \\
\text { ème siècle }\end{array}$ & $\begin{array}{l}\text { Vision éthérée, indéfinissa- } \\
\text { ble et romantique d'un autre } \\
\text { imaginé }\end{array}$ \\
\hline
\end{tabular}

${ }^{23}$ Willberger (1972) et Wolff (1994).

${ }^{24}$ Davies (2006)

${ }^{25}$ Feher (1988).

${ }^{26}$ Davies (2001: 375-378)

${ }^{27}$ Voir Hammond (2004), Haynes (2004) et Todorova (1997).

${ }^{28}$ Cardini (2000), Corm (1989, 2002, 2009) et Saïd (1980). 


\section{Mauvais concepts - mauvaise politique}

La dissonance cognitive dont souffre la construction européenne rend difficile de la penser. Trois exemples mettent en exergue cette tâche compliquée.

\subsection{Les échecs}

\section{L'élargissement}

L'élargissement est une des causes de la fatigue communautaire. Dix pays ont rejoint l'UE, transformant radicalement ses frontières, sa géographie mentale et son échelle. L'UE est passée à cinq cents millions d'habitants, repoussant ses limites jusqu'à l'ancienne URSS, la Turquie et les Balkans. Selon Christian Lequesne (2007 et 2008), le choc intellectuel a été terrible. On ne pouvait soudain plus penser la construction européenne uniquement et exclusivement en termes d'Europe de l'Ouest fondée sur la carte imaginaire de l'Empire de Charlemagne. ${ }^{29} \mathrm{Il}$ a fallu changer de paradigme pour penser l'UE après 1989, mais les tenants de l'Europe politique ont été d'autant plus rétifs à le faire que l'élargissement était perçu comme une concession aux défenseurs du grand marché européen et du libéralisme. L'Europe étant jusque-là pensée selon la dichotomie approfondissement/élargissement, ce dernier semblait se faire au détriment du premier voire mettre en danger la totalité de l'édifice, au moment même où l'on achevait de mettre en place le marché unique et où l'on coulait les fondations de l'union économique et monétaire. C'était aussi mettre aux oubliettes le référentiel d'une construction européenne focalisée sur le noyau dur franco-allemand.

Surtout, la perception des nouveaux pays membres ne rendait pas la tâche plus facile, surtout du point de vue français - si important ultérieurement, du fait du rejet du Traité constitutionnel européen. Considérés comme un bloc indivis de jungles néolibérales adeptes du dumping social et des délocalisations, (trop) fortement atlantistes et antirusses, plus enclines à écouter Washington que Bruxelles, les nouveaux pays membres sont restés méconnus et méconnaissables précisément parce qu’on leur appliquait des préjugés résultant d'une écriture biaisée de l'histoire. Lequesne en tire une conséquence pra-

\footnotetext{
${ }^{29}$ Le regretté professeur Geremek avait pour habitude de poser à ses étudiants du Collège d'Europe la question de savoir si l'empire carolingien pouvait être une référence pour la construction européenne. Le mythe fondateur de l'Europe chrétienne des six et de la réconciliation franco-allemande ont été mis à rude épreuve avec l'élargissement de l'UE aux anciens pays barbares.
} 
tique et réaliste : l'apparition d'une anxiété vis-à-vis des nouveaux membres et de leurs avantages comparatifs fondés sur un moindre coût de la main-d'œuvre dans un contexte de morosité économique et sociale.

\section{Migration, Traité constitutionnel européen et marché du travail}

Dans ce contexte, le nationalisme et le protectionnisme latents ont été activés par le catalyseur que s'est révélé être le référendum sur le Traité constitutionnel. Celui-ci a aussi aidé à cristalliser le débat sur la Directive Bolkenstein et le fantasme du plombier polonais (voir Caune et alii, 2007). En ce sens, le débat sur le TCE a remplacé celui qui n'a pas eu lieu sur l'élargissement, qui suscitait aussi bien les angoisses de l'absorption de dix nouveaux pays membres venant de cette région impensée qu'est l'Est, que l'anxiété d'un élargissement donnant raison au libéralisme (on retrouve ici la dichotomie approfondissement-élargissement).

L'histoire du plombier polonais, durant le référendum sur le TCE, met en lumière les difficultés à penser la nouvelle Europe élargie dans un concept inclusif. La fausse représentation d'une dichotomie approfondissement-élargissement a fait le lit d'une contestation antilibérale fondée sur des préjugés. Ceux-ci ont été activés par le fantôme d'un élargissement à une Europe libérale et la discussion simultanée de la Directive Bolkenstein. Mais comment le plombier polonais est-il apparu? Dans un article du Figaro du 15 mars 2005, Philippe de Villiers a brocardé la directive en question et s'est saisi de ce prétexte pour se positionner sur le sujet du TCE. Ainsi, selon lui, « la Directive Bolkenstein permet à un plombier polonais ou à un architecte estonien de proposer ses services en France, au salaire et avec les règles de protection sociale de leur pays d'origine. Sur les onze millions de personnes actives dans les services, un million d'emplois sont menacés. Il s'agit d'un démantèlement de notre modèle économique et social ». On peut s'interroger sur les prémisses d'une telle affirmation péremptoire. Premièrement, cela suppose une attractivité du marché du travail français. On peut en douter vu le faible taux d'emploi et l'important taux de chômage. C'est d'ailleurs confirmé a posteriori par le nombre de polonais en France, nettement inférieur à celui de polonais en Grande-Bretagne et à l'Irlande. Deuxièmement, cela implique que les travailleurs de l'Est veuillent émigrer dans une contrée hostile et inconnue, ce qui n'est pas si facile, et qu'ils puissent le cas échéant se satisfaire d'une protection sociale inexistante. On ne saurait avoir une vision plus condescendante de ses voisins de l'Est, toujours perçus comme des pauvres «barbares » dans les jardins prolifiques de l'Occident. C'est aussi méconnaître profondément les réalités des populations vivant dans la pénurie perma- 
nente depuis plus de soixante ans. Peut-on émigrer dans un pays plus riche et y travailler sans attendre en retour les mêmes services que ses concitoyens ?30

Une analyse statistique du vote français (Brouard et alii, 2007) souligne que les variables dominantes du vote ont été le caractère anxiogène de la mise en cause du modèle social français et un euroscepticisme condamnant une UE à la rhétorique libérale. L'élargissement, s'il a influencé le vote notamment par les frustrations de n'avoir aucun contrôle sur l'entrée de l'ancienne « Europe de l'Est » et la peur d'un élargissement incontrôlable à la Turquie, s'est révélé une variable indirectement décisive du vote sur le Traité Constitutionnel. Ces élargissements perçus comme illégitimes ont activés une contestation sociale latente qui explique la progression de l'euroscepticisme à gauche (sans doute la grande nouveauté politique suscitée par le TCE).

\section{La crise financière et sa propagation à l'Est}

La contagion de la crise financière offre une autre preuve du manque dramatique de différentiation des pays de l'Est aux yeux de l'Occident. Dans le sillage de la propagation à l'Europe et des conditions difficiles des économies hongroises et ukrainiennes, les investisseurs ont retiré subitement tous leurs capitaux en «appuyant sur une touche de leur clavier - Europe Centrale et Orientale », comme le rapporte l'hebdomadaire polonais Polityka. ${ }^{31}$

Cette propagation repose sur deux mécanismes. D'abord le cercle herméneutique dont les préjugés ont empêché de différentier les pays de l'Est. Une fois l'article du Financial Times publié ("The East is in the Red», 16 octobre 2008), ce sont tous les financiers de la City qui ont retiré leurs avoirs de peur de voir l'Est s'effondrer à son tour. Ce mouvement a été renforcé par une série d'articles publiés dans les médias les plus lus. ${ }^{32}$ Ensuite, un deuxième mécanisme s'est mis en branle, presque simultanément. Il s'agit de ce que le sociologue Américain Robert Merton (1968) a appelé « la prophétie autoréalisatrice » ou « l'effet d'Oedipe » selon l'épistémologue Karl Popper (1976). Ces deux expressions renvoient à la réalisation paradoxale d'une assertion erronée. Une définition de la situation d'abord fausse provoque un comportement qui finit par la valider parce que ladite définition devient un élément de la situation et peut donc l'influencer. Dans

\footnotetext{
${ }^{30}$ Pour des études mettant en relation perception des migrations et marché de l'emploi dans l'UE, voir Beutin et al. (2007) et Canoy et al. (2009).

${ }^{31}$ «Węgierski syndrom » (Le syndrome hongrois), 18 octobre 2008.

32 Parmi lesquels: «A Chill Wind Blows East », The Economist, 17 octobre 2008; « Call for West Europe to Support East », Financial Times, 20 octobre 2008; «Who's Next? », The Economist, 23 octobre 2008; «Crisis Tests Stability of New EU States », Financial Times, 15 janvier 2009.
} 
le cas de la propagation de la crise à l'Est, une fausse information sur l'état des économies est-européenne peut créer une crise dans toute la région.

Alors qu'il n'y avait que la Hongrie, la Lettonie et l'Ukraine à se trouver dans des situations instables, ce sont toutes les devises et les bourses de la région qui ont dévissé. Cela en dépit d'une situation économique relativement saine en Pologne et en République tchèque. Même chose pour les États baltes. Leur situation est diamétralement opposée: la Lettonie a, comme la cigale, subi la crise de plein fouet tandis que l'Estonie, qui a mené une politique fiscale solide durant les dernières années, a pu faire face à la crise avec une certaine marge de manœuvre. Pour qui veut observer l'évolution de la situation dans la région depuis plusieurs mois, ces pays essayent d'enrayer les deux mécanismes du cercle herméneutique et de la prophétie autoréalisatrice en cherchant à se démarquer les uns des autres. La Pologne a vite annoncé sa volonté de rejoindre la zone euro et de contrôler son déficit. L'Estonie a proposé une aide financière à la Lettonie pour prouver qu'elle n'avait pas besoin de l'aide du Fonds monétaire international et afin d'atténuer tout mouvement à la baisse des marchés et de sa devise (d'autant plus important que la couronne estonienne fait de facto partie du mécanisme du taux de change européen).

On voit donc les conséquences pratiques de jugements fondés sur des préjugés inadéquats. Les interprétations intellectuelles d'une situation, quelle que soit leur pertinence, ont des conséquences réelles sur la situation.

\subsection{La difficulté à penser l'Europe de l'Est}

Ces erreurs politiques amènent à s'interroger sur les concepts dont l'on se sert pour penser les pays d'Europe de l'Est. Il y a comme un gouffre qui apparait dès que l'on observe les réalités de l'Europe de l'Est et les concepts utilisés pour les décrire.

Les particularités de l'Europe de l'Est sont en effet souvent insaisissables par la manière de voir occidentale. Pomian (1991) montre les conséquences de la spécificité de l'emplacement géographique de cette Europe «peuplée des nations dont chacune, majoritairement catholique ou protestante, a été, pendant des décennies, voire des siècles, en relation de voisinage territorial » avec des nations majoritairement orthodoxes ou sous influence musulmane. L'Europe de l'Est est précisément cet entre-deux situés dans l'espace mouvant à la frontière de l'ancien Empire Carolingien et celle du Schisme de 1054 qui a donné lieu à des développements idiosyncratiques décrits par Szücs (1985). Cette structure s'est bâtie sur des discontinuités en perpétuelle évolution (loin du cadre plus stable mais changeant de l'Occident). Ces discontinuités sont à trouver dans une myriade de systèmes politiques entretenant des rapports uniques et différents avec le religieux, l'économie, l'intégration nationale à géométrie variable du point de vue ethnique, linguistique, culturel ou religieux - mais pas, selon Pomian (1991) avec l'État. La catégorie de l'État centralisé est inapplicable à ces régions qui furent longtemps soit des empires multiethniques, multilingues et multiculturels, soit des populations sujettes de 
la domination des États voisins. Ces systèmes sont, en effet, la proie permanente de la compétition entre les deux pôles qui l'encerclent. Comment par exemple appliquer la catégorie d'État à des pays comme le Belarus, l'Ukraine, la Slovaquie, etc. ? Une intéressante étude de Snyder (2004) reconstruit une histoire de l'Europe du centre-est non pas de la manière occidentale, c'est-à-dire en narrant l'évolution de l'État-Nation depuis le Moyen-âge jusqu'à nos jours, mais en se concentrant sur l'émergence de l'idée nationale de quatre contrées (la Pologne, la Lituanie, l'Ukraine et le Belarus) à partir d'un empire multiethnique (l'Union Polono-Lithuanienne). On découvre en filigrane que des notions comme l'identité homogène ou l'État sont pratiquement inopérantes pour rendre compte du processus complexe qui a mené de l'un à l'autre et que l'idée de nation est plus compliquée que son sens immédiat en Occident.

Ainsi, les questions que pose l'histoire de cette « autre Europe » sont radicalement différentes de celles de l'Europe de l'Ouest. On peut rassembler les questions relatives à l'Europe de l'Est (voir tableau 3) en s'appuyant sur Bibo (1986) ainsi que Comisso et Gutierrez (2002). Si l'on compare les concepts majeurs de la littérature consacrée à la région (tableau 4), on se rend compte du hiatus conceptuel.

\section{Tableau 4. Les concepts de « l'autre Europe»}

\begin{tabular}{|c|c|}
\hline Concept & Description \\
\hline la question de l'État & $\begin{array}{l}\text { État multiethnique, décentralisé } \\
\text { État distinct de sa société } \\
\text { dans le cas d'occupation étrangère }\end{array}$ \\
\hline la formation de la nation & nations multiculturelles \\
\hline construction de l'identité & identités complexes \\
\hline économie & $\begin{array}{l}\text { économie reprenant soumise à la fragmentation des } \\
\text { discontinuités historiques et géographiques }\end{array}$ \\
\hline objet des relations internationales & $\begin{array}{l}\text { non souveraineté, dépendance des empires limitro- } \\
\text { phes }\end{array}$ \\
\hline
\end{tabular}

Deux exemples peuvent aider à comprendre ce hiatus conceptuel. Le premier est celui de l'Ukraine et les études qu'elle a suscitées. La chute de l'empire communiste a fait émerger sur la carte de l'Europe centrale et orientale des pays longtemps disparus. Parmi eux, l'Ukraine, dont l'apparition a scellé la fin de l'Empire russe selon Zbigniew 
Brzeziński, a fait l'objet d'analyses en termes en termes de démocratisation et de régime politique autoritaire. Cette démarche allait de soi dans la mesure où les observateurs occidentaux attendaient de l'ancien bloc de l'Est qu'il se réforme à l'image des démocraties et des économies de marché occidentales. C'était l'objet d'un livre brillant décrivant les critères en vertu desquels on pouvait juger de la démocratisation des pays sudaméricains et post-soviétiques (Linz et Stepan, 1996). Cette approche a semblé être confirmée par la Révolution orange. Or Stepan (2005) s'est rendu compte que, dans le cas ukrainien, cette approche passait sous silence les tensions profondes de la société ukrainienne et qu'il tenait pour acquise la notion d'État, évidente en Occident. Les analyses ont fait l'économie du problème du demos et de l'ethnos ukrainiens, condition de possibilité de la démocratie en Ukraine. La question de l'identité ukrainienne a été mise de côté. Or, qu'est ce qu'une démocratie sans le peuple qui la constitue? Le manque de cette donnée a conduit à des impasses intellectuelles où l'on cherchait à juger d'après des critères objectifs mais aveugles une progression impossible sans le fondement qu'elle présuppose (Ryabtchouk, 2003).

Le second exemple concerne le nationalisme. Davies (2006: 30-32) décrit comment l'étude du nationalisme diffère selon qu'il s'agit de l'Ouest ou de l'Est. Citant des historiens renommés comme Plamenatz, Ernst Gellner et d'autres, il met en évidence les préjugés de l'étude du nationalisme. Plamenatz prenait en effet une optique culturaliste pour définir les différents types de nationalisme. D'un côté, le nationalisme de l'Ouest est « bien équipé culturellement » avec une langue « adaptée », des universités, des écoles et une civilisation progressive. Cela concerne les nationalismes italien, allemand, français ou anglais, et rappelle la construction intellectuelle de la civilisation occidentale. La clef de voûte de cette construction nationale est à trouver dans l'émergence d'un État fort cherchant à faire émerger une identité nationale - le plus souvent celle du centre au détriment de celle des périphéries. De l'autre côté, le nationalisme slave que Plamenatz qualifie de non-européen. Il renvoie à des tentations de création d'une identité nationale indépendamment de l'État - comme dans le cas est européen sous la domination des empires russe, turc, allemand et austro-hongrois. Dans les deux cas, le concept de nationalisme renvoie à deux réalités différentes. Mais il est aussi malheureusement chargé de deux connotations différentes. 
Tableau 5. Concepts utilisés pour analyser «l'autre Europe »

\begin{tabular}{|c|c|c|c|}
\hline Concept & Période & Description & Limite à l'Est \\
\hline État & Moderne & $\begin{array}{l}\text { Centralisation d'un pouvoir } \\
\text { absolu et monopoliste }\end{array}$ & \multirow{2}{*}{$\begin{array}{l}\text { Période d'empires } \\
\text { multiethniques en Eu- } \\
\text { rope centrale et de } \\
\text { monarchies électives }\end{array}$} \\
\hline Nation et nationalisme & $19 \mathrm{e}-20^{\mathrm{e}}$ & $\begin{array}{l}\text { Homogénéisation des popu- } \\
\text { lations et d'une identité }\end{array}$ & \\
\hline Totalitarisme & \multirow{5}{*}{ 1945-1989 } & \multirow{5}{*}{$\begin{array}{l}\text { Approche d'un objet de re- } \\
\text { cherche par différents para- } \\
\text { digmes explicatifs }\end{array}$} & \multirow{5}{*}{$\begin{array}{l}\text { Caractère contingent } \\
\text { et anhistorique des } \\
\text { analyses ignorant la } \\
\text { société civile, les évo- } \\
\text { lutions du système so- } \\
\text { cialiste et l'hétérogé- } \\
\text { néité du camp } \\
\text { socialiste. URSS- } \\
\text { centré. }\end{array}$} \\
\hline Classe dominante & & & \\
\hline Système communiste & & & \\
\hline Groupes politiques & & & \\
\hline Bureaucratie & & & \\
\hline Post-socialisme & 1989-2004 & $\begin{array}{l}\text { Evolution après la chute du } \\
\text { bloc de l'Est }\end{array}$ & \multirow{4}{*}{$\begin{array}{l}\text { Contingent et téléolo- } \\
\text { gique }\end{array}$} \\
\hline Transition & 1989-2004 & \multirow{2}{*}{$\begin{array}{l}\text { Mis en place de régimes } \\
\text { démocratiques et d'écono- } \\
\text { mies de marché }\end{array}$} & \\
\hline Démocratisation $^{33}$ & $\begin{array}{l}1989 \text { à nos } \\
\text { jours }\end{array}$ & & \\
\hline Élargissement & $\begin{array}{l}2004 \text { à nos } \\
\text { jours }\end{array}$ & Impact de l'entrée dans l'UE & \\
\hline Européanisation & $\begin{array}{l}2004 \text { à nos } \\
\text { jours }\end{array}$ & $\begin{array}{l}\text { Transformation par le haut } \\
\text { et homogénéisation par des } \\
\text { normes «meilleures » }\end{array}$ & $\begin{array}{l}\text { Voile d'ignorance sur } \\
\text { les idiosyncrasies et les } \\
\text { stratégies d'adaptation }\end{array}$ \\
\hline Centre/périphérie & $19 \mathrm{e}-20^{\mathrm{e}}$ & $\begin{array}{l}\text { Explique la relation entre un } \\
\text { centre développé et une pé- } \\
\text { riphérie sous-développée et } \\
\text { dépendante du centre }\end{array}$ & $\begin{array}{l}\text { Jugement de valeur } \\
\text { implicite et hiérarchi- } \\
\text { sation }\end{array}$ \\
\hline
\end{tabular}

${ }^{33}$ Linz et Stepan (1996). 
La difficulté à penser cette « autre Europe » apparemment insaisissable vient donc de cette incapacité à surmonter les préjugés de l'Occident induits en partie par le rapport de force historique et par les outils conceptuels inadaptés à la réalité est-européenne.

\section{Conclusion}

Que pèsent les perceptions et les préjugés? L'étude du cas Est-Ouest et des structures de compréhension utilisée par l'Ouest à l'égard de l'Est montre que les préjugés et les perceptions ont une grande influence sur la compréhension - vraie ou fausse - que l'on peut avoir d'une région. Le cercle herméneutique est un cadre analytique idoine pour déconstruire les échecs intellectuels et politique de l'Union européenne élargie. Les attentes crées par des concepts biaisés ont engrangés un cercle herméneutique qui a oblitéré la complexité et l'altérité de l'Europe de l'Est. Une géographie mentale s'est esquissée qui, fondée sur une imaginaire inadéquat, a conduit à des erreurs politiques majeures.

Le plus intéressant est sans doute l'émergence dans les dernières décennies d'études approfondies menant la vie dure aux représentations de « l'autre Europe ». Encore plus intéressant est néanmoins l'échec relatif de ces études à se propager dans les opinions et les cartes mentales. Il y a comme un impensé indélébile dans la conscience européenne. Il provient de la permanence d'une conceptualisation exclusive de l'Union européenne et qui fait parler d'une « Europe à deux vitesses ».

S'il y a donc bien une tâche à laquelle doit s'atteler la jeune génération d'Européens, c'est bien de repenser l'Europe tel quelle est et telle qu'ils la désirent, et non plus selon des concepts qui ont déjà montré leur inadéquation à la situation présente. Mais cela est une autre histoire.

\section{Bibliograhie :}

Beutin, Ricklef; Canoy, Marcel; Horvath, Anna; Hubert, Agnès; Lerais, Frédéric et SochackI, Myriam, «Reassessing the Link between Public Perception and Migration Policy », in European Journal of Migration and Law, 9(4), 389-418.

BIBO, Istvan (1986), Misère des petits États d'Europe de l'Est, L'Harmattan, Paris.

Brouard, Sylvain, Grossman, Emiliano, SAuger, Nicolas (2007), Les Français contre l'Europe, Presses de Sciences Po.

Canoy, Marcel; Horvath, Anna; Hubert, Agnès; Lerais, Frédéric et Sochacki, Myriam (2009), "Post-Enlargement Migration and Public Perception in the European Union », in KAHANEC M. and Zimmermann K. F. (eds.), EU Labor Markets after Post-Enlargement Migration, Springer: Berlin.

CARDINI, Franco (2000), Europe et Islam. Histoire d'un malentendu, Paris, Seuil. 
CASTORIADis, Cornelius (1999), L'institution imaginaire de la société, Paris, Seuil.

Caune, Hélène, Lemaire-Dujin, Anne, Petithomme, Mathieu et Truchlewski, Zbigniew (2007), «Les débats suscités par le Traité constitutionnel en France: illustration, cristallisation et déplacements des clivages traditionnels », Notes et études de l'Unité de Science politique et de relations internationales (SPRI), Université Catholique de Louvain, numéro 2.

Comisso, Ellen and GutTIERrEZ, Brad (2002), « Eastern Europe or Central Europe? Exploring a distinct regional identity », UCIAS, Vol. 3, article 7.

COMMISSION EUROPEENNE (2008), Rapport de 2008 sur l'impact de la libre circulation des travailleurs dans le contexte de l'élargissement de l'UE de l'UE [COM(2008)765 final], Bruxelles, novembre.

CORM, Georges (1989), L'Europe et l'Orient: de la balkanisation à la libanisation, bistoire d'une modernité inaccomplie, Paris, La Découverte.

CORM, Georges (2002), Orient-Occident. La fracture imaginaire, Paris, La Découverte.

CORM, Georges (2009), L'Europe et le mythe de l'Occident. La construction d'une histoire, Paris, La Découverte.

DAVIES, Norman (1996), Europe : A History, Oxford : Oxford University Press.

Davies, Norman (2001), Heart of Europe: The Past in Poland's Present, Oxford University Press, USA.

DAVIES, Norman (2006), Europe East \& West, Jonathan Cape.

DeHousse, Renaud et alii (2007), Elargissement: Comment l'Europe s'adapte, Presses de Sciences Po.

DiLTHEY, Wilhem (1995 [1900]), «La naissance de l'herméneutique », in CEuvres, tome 7, Paris, Cerf.

DMITRIEVA, Ekaterina, «Visions russes de l'Europe centrale: occidentalistes et slavophiles », Revue Germanique Internationale, $\mathrm{n}^{\circ} 1$.

FAuve, Adrien (2007), «Astana, nouvelle capitale du Kazakhstan : entre mythe et réalités », in Regards Sur l'Est, No. 47, Novembre.

FEHER, Ferenc (1988), « Eastern Europe's Long Revolution Against Yalta », East European Politics and Societies, Vol. 2, No. 1, Winter, pp. 20-41.

Gadamer, Hans Georg (1996 [1960]), Vérité et Méthode, Editions du Seuil, Paris.

GARTON AsH, Timothy (1999), "Where is Central Europe now?", in History of the Present, Essays, Sketches, and Dispatches from Europe in the 1990s, Allen Lane. 
GODSWORTHY, Vesna (1998), Inventing Ruritania: the Imperialism of the Imagination, New Haven and London: Yale University Press.

HALL, Edith (1989), Inventing the Barbarian: Greek Self-Definition through Tragedy, Oxford Classical Monographs.

HAMmOND, Andrew (2004), "Introduction», The Balkans and the West: Constructing the European Other 1945-2003, Ashgate.

HAYNES, Michael (2004), «The Rhetoric of Economics : Cold War Representation of Development in the Balkans ", in The Balkans and the West: Constructing the European Other 1945-2003, Andrew Hammond (ed.), Ashgate.

HeIDEGGER, Martin (1986 [1927]), Etre et Temps, §32, Gallimard, Paris.

HERGE, Le Sceptre d'Ottokar, 1939.

HERGE, L'Affaire Tournesol, 1956.

Iordanova, Dina (2001), Cinema of Flames: Balkan Films, Culture and the Media, London : BFI.

JUDT, Tony (1991), "The Rediscovery of Central Europe », in GRAUBARD, Stephen R. (dir.), Eastern Europe, Central Europe, Europe..., Boulder, San Francisco, Oxford.

KULCZYCKI, John J. (2005), «Eastern Europe in Western Civilization Textbooks : the Example of Poland », The History Teacher, 38, 2, pp. 153-177, February.

KONRAD, Gyorgy (1994), The Melancholy of Rebirth, New York: Harcourt Brace.

KunderA, Milan (1984), "The Tragedy of Central Europe », in New York review of Books, 26 April.

LACOSTE, Yves (1988), «Editorial: Europe médiane ? », Hérodote, 48, 1 ${ }^{\text {er }}$ Trimestre, pp. 3-12.

LEQUeSNE, Christian (2007), « Penser l'Europe élargie : les carences du débat français », Le Monde, 8 novembre.

LEQUeSne, Christian (2008), La France dans la Nouvelle Europe: Assumer le changement d'échelle, Presses de Sciences Po, Paris, 2008.

LinZ, Juan et STEPAn, Alfred (1996), Problems of Democratic Transition and Consolidation, Baltimore, Johns Hopkins University Press.

MARES, Antoine (1991), « La vision française de l'Europe centrale, d'un prisme à l'autre, du XIX ${ }^{\text {ème }}$ au XX ${ }^{\mathrm{ème}}$ siècle », in BEAUPETRE, Gérard, L'Europe centrale. Réalité, mythe, enjeu, Varsovie.

MERTON, Robert (1968), Social Theory and Social Structure, New York: Free Press. p. 477. 
MeYer Cord Henry (1995), Mitteleuropa in German Tought and Action 1815-1945, La Haye.

Michel, Patrick (dir.) (1997), L'Europe médiane. Au seuil de l'Europe, Paris.

NAUmann Friedrich (1915), Mitteleuropa, tr. de l'allemand par l'Argus suisse de la Presse a Geneve, Neuchatel et Paris, Delachaux \& Niestlé - Payot.

Pomian, Krzysztof (1991), "Les particularités historiques de l'Europe Centrale et Orientale », Le Débat, 63, janvier-février, pp. 26-40.

POPPER, Karl (1976), Unended Quest: An Intellectual Autobiography, LaSalle, IL: Open Court.

Rosslyn, Felicity (2004), "Primitivism and the Modern: A Prolonged Misunderstanding ", in The Balkans and the West: Constructing the European Other 1945-2003, Andrew Hammond (ed.), Ashgate.

RUPNIK, Jacques (1990), L'autre Europe, crise et fin du communisme, Paris, Odile Jacob / Points Seuil.

RYABTCHOUK, Mykola (2003), De la «Petite Russie » à l’Ukraine, L'Harmattan, Collection Présence Ukrainienne.

SAID, Edward (1980), L’Orientalisme : l'Orient créé par l'Occident, préf. de Tzvetan Todorov ; trad. de l'américain par Catherine Malamoud, Paris : Seuil.

SINNHUBER, Karl A. (1954), «Central Europe - Mitteleuropa - Europe Centrale : An Analysis of a Geographical Term », Institute of British Geographers Transactions and Papers.

SNYDER, Timothy (2004), The Reconstruction of Nations: Poland, Ukraine, Lithuania, Belarus, 1569-1999, New Haven and London: Yale University Press.

STEPAN, Alfred (2005), "Ukraine : Improbable Democratic "Nation-State" But Possible Democratic "State-Nation" », in Post-Soviet Affairs, 21, 4, Décembre.

SyMANIEC, Virginie (1997), Parlons biélorussien, Ed. L'Harmattan.

SzUCS, Jeno (1985), Les Trois Europes, tr. fr. du hongrois par Véronique Charaire, Gábon Klahiczay et Philippe Thureau-Dangin, Paris, L'Harmattan.

Todorova, Maria (1991), "Myth Making in European Family History: the Zadruga Revisited », East European Politics and Society, 4 (1) ; pp. 30-69.

Todorova, Maria (1997), Imagining the Balkans, Oxford University Press, New York.

TODOROVA, Maria (2003), "Learning memory, remembering identity ", in Balkan Identity : Nation and Memory, Todorova, Maria (ed.), Hurst, London and New York University Press. 
VACAR, Nicholas (1949), "The Name "White Russia” », in The American Slavic and East European Review, Vol. 8, No. 3.

WeST, Rebecca (1941), Black Lamb And Grey Falcon: A Journey Through Yugoslavia, Penguin Books.

Willberger, Carolyn H. (1972), Voltaire's Russia: Window on the East, Oxford University Press.

WolfF, Larry (1991), «Rebecca West: This Time, Let's Listen », New York Times, OpEd, 10 February.

WolfF, Larry (1994a), « "If I Were Younger I Would Make Myself Russian" : Voltaire’s Encounter With the Czars », New York Times, Op-Ed, 13 November.

WolfF, Larry (1994b), Inventing Eastern Europe: The Map of Civilization on the Mind of the Enlightenment, Stanford University Press.

WolfF, Larry (2001a), «A New Mental Map of the World : Dalmatia, the Eastern Bloc, and the Seacoast of our Imagination », New York Times, Op-Ed, 28 June 2001.

WolfF, Larry (2001b), Venice and the Slavs: The Discovery of Dalmatia in the Age of Enlightenment, Stanford University Press.

Zaprudnik, Jan (1993), Belarus: At a Crossroads in History, Westview Press.

ZrinsCAK, Geogette (1999), L'Europe Médiane: des pays Baltes aux Balkans, Paris, La Documentation Française (Dossier no 8005). 